\title{
NASA GRC UAS Project \\ Communications Modeling and Simulation Development Status
}

\author{
Presenter - Rafael Apaza \\ NASA Glenn Research Center \\ 21000 Brookpark Rd. \\ Cleveland, Ohio 44135
}

\section{Modeling and Simulation Team Contributors}

Steve Bretmersky / Justin Dailey - MTI Systems

Goutam Satapathy / David Ditzenberger / Chris Ye - Intelligent Automation Inc.

Greg Kubat / Christine Chevalier / Thanh Nguyen - Vantage Partners, LLC.

Rafael Apaza (Lead) - NASA GRC

2014 Integrated Communication, Navigation, and Surveillance (ICNS) Conference

8-10 April 2014

Herndon, Virginia 
GRC UAS Project - Communications Modeling and Simulation GRC UAS Communications Modeling - Objectives

\section{Primary Objectives}

Use Modeling and Simulation to perform analysis to support recommendations for integration of UAS CNPC systems for safe and efficient operation of UAS in the NAS.

Support CNPC Datalink MOPS Development

Defined Two Primary Development Activities to meet these objectives

- Develop Models of Radios and Comm System Architecture for UAS CNPC Radio datalink operations

- Develop Large-scale, NAS-wide Simulation capability with NAS ATM Communications and integrated UAS CNPC Communications 


\section{GRC UAS Project - Communications Modeling and Simulation}

\section{GRC UAS Communications - Mod/Sim Approach}

\section{Develop Models of GRC CNPC Flight Test Radios using Opnet Modeler \\ - Test, Verify and Validate Model operation \\ - Perform simulations to study detailed radio performance \\ - Assist with Radio/Radio system design}

Integrate Opnet Radio models into Large-scale Simulation Architectures

\section{Develop UAS Comm Architecture simulation capability in NASA 'ACES'}

- ACES provides NAS CONUS Airspace Operations for Flights and Legacy ATM Communications Models

- Develop One Relay and One Non-Relay UAS Comm Architecture model in ACES

\section{Combined ACES-Opnet simulations:}

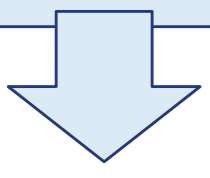

- Provide interoperability between NAS Airspace operations, NAS air traffic loads, UAS Air traffic loads, existing NAS ATM Comm and new CNPC UAS Comm Systems

- Allow us to characterize NAS Comm Message Traffic levels that will exist with UAS

- Allows for studies of ATM Comm delay and latency data in UAS Comm architectures

- Allows us to evaluate NAS ATM Comm Performance and CNPC link performance in a common simulation environment. 


\section{GRC UAS Project - Communications Modeling and Simulation GRC UAS Communications - Opnet CNPC Radio Models Description}

CNPC Opnet models are being developed to:

- Support a scalable, networked CNPC topology that allows BLOS operations

- Simulate the prototype and future CNPC radio protocol operations

- Physical layer transmission/reception timing, frequency, data rate, and bandwidth

- Connection establishment and handoff operations

- Data transfer including traffic prioritization and queuing

- Model a relevant RF propagation environment for UA operations

- Includes terrain effects

- Detailed models will be derived from an extensive channel sounding study

Outputs of the CNPC model allow analysis of:

- Throughput and delay of C2 and relay data throughout a flight

- RF performance and health estimates at all points in a flight profile

- Usage and loading for CNPC towers for a set of flights

- Tower deployment scenarios

- Cell radius affecting tower spacing and total number of towers

- Frequency reuse plans 
GRC UAS Project - Communications Modeling and Simulation GRC UAS Communications - Opnet CNPC Gen1 Radio Model Development

\section{Gen1 Model Development}

- The Gen1 model focused on implementing the waveform basics

- TDD with TDMA uplink and FDMA downlink

- Simple non-reliable data link protocol that supports queuing and segmentation

- Designed to support single aircraft / single tower scenario

- Layer-3 and above protocols are abstracted

- Implementation and testing completed 4/2013

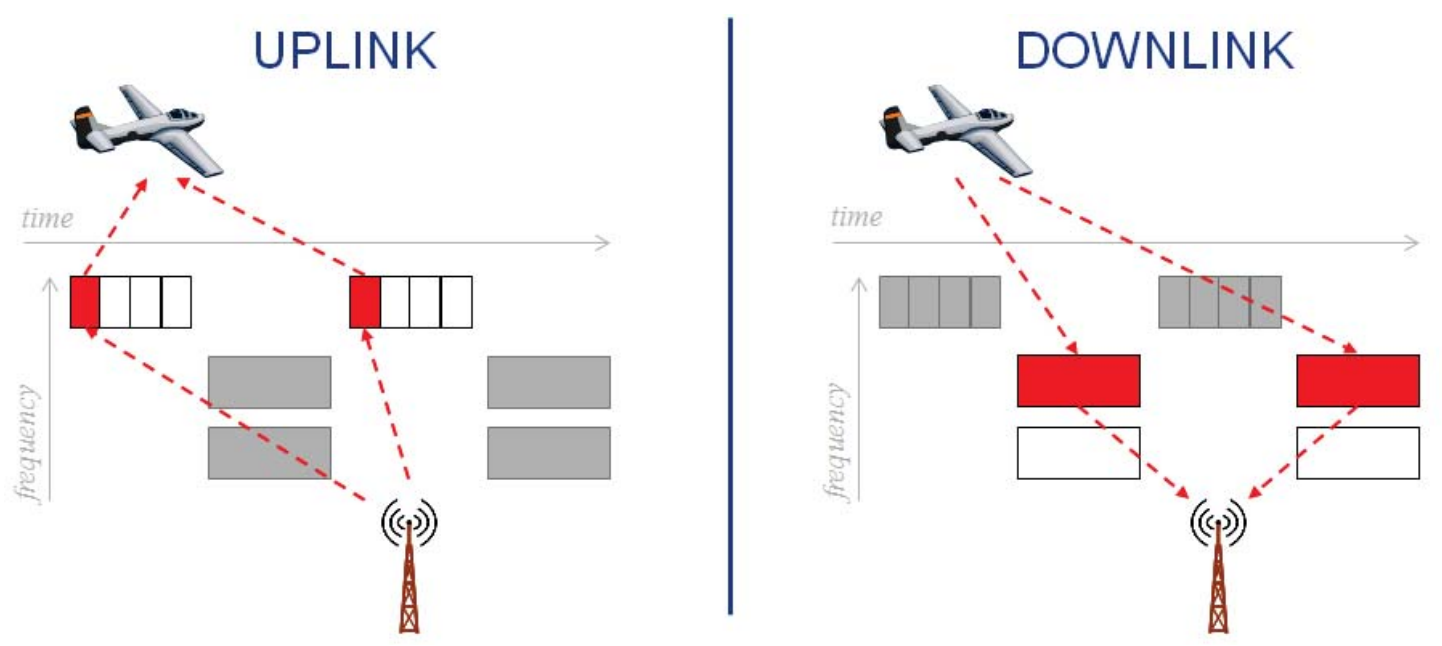


GRC UAS Project - Communications Modeling and Simulation GRC UAS Communications - Opnet CNPC Gen2 Radio Model Development

\section{Gen2 Model Development}

- The Gen2 model expands on the waveform

- Supports multiple data link connections with priority

- Adds bi-directional link health assessment

- Allows for simple handoff scenarios

- Designed to support single aircraft / two-tower scenario like prototype, with multiple aircraft / multiple tower enhancements to start larger-scale simulations

- Layer-3 and above protocols are still abstracted

- Implementation in progress, testing to be completed 7/2014

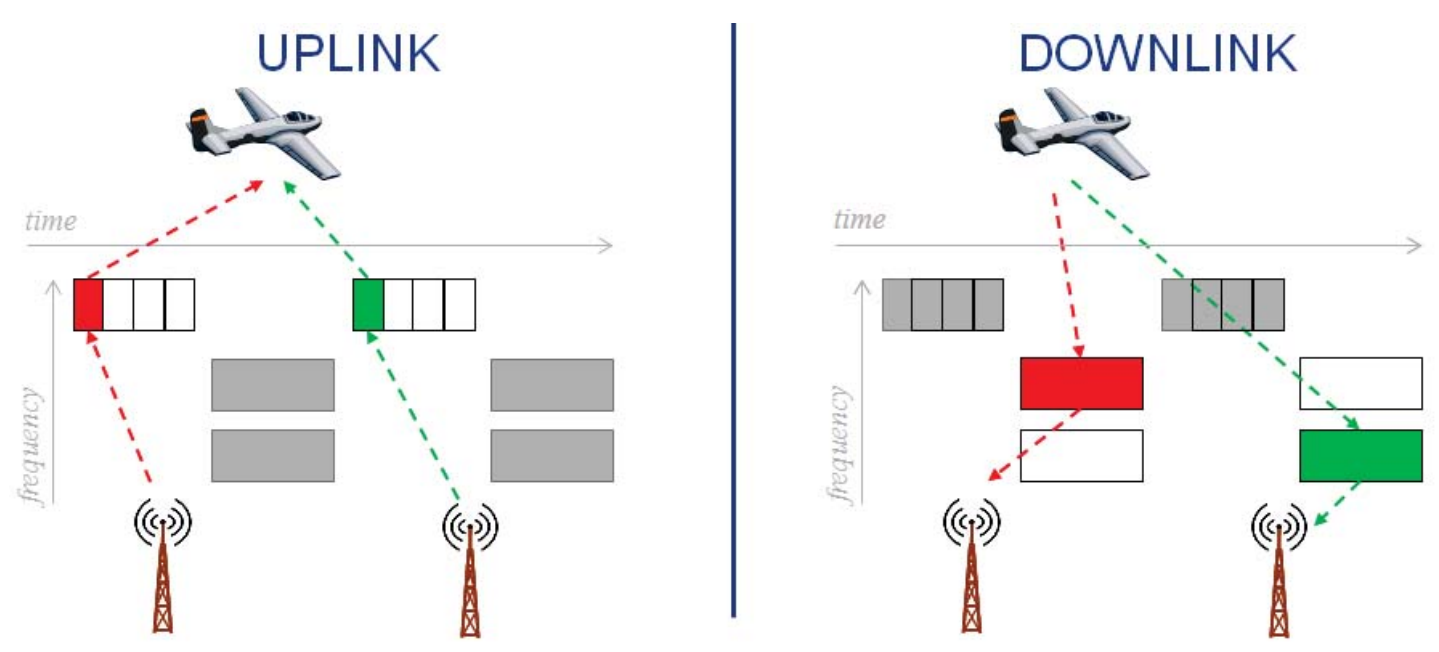


GRC UAS Project - Communications Modeling and Simulation GRC UAS Communications - Future Opnet CNPC Radio Model Development

\section{Future Model Development}

- Future modifications to the model will further enhance its capabilities

- Implement system ingress procedures

- Expands handoff functionality

- Support reliability in the data link protocol

- Designed to support multiple aircraft / multiple-tower scenarios like Gen3 and later prototypes

- Add Layer 3 and 4 models

- TCP/UDP over IPv6

- Research refinements to RF model

- Include 2-ray and diffraction effects
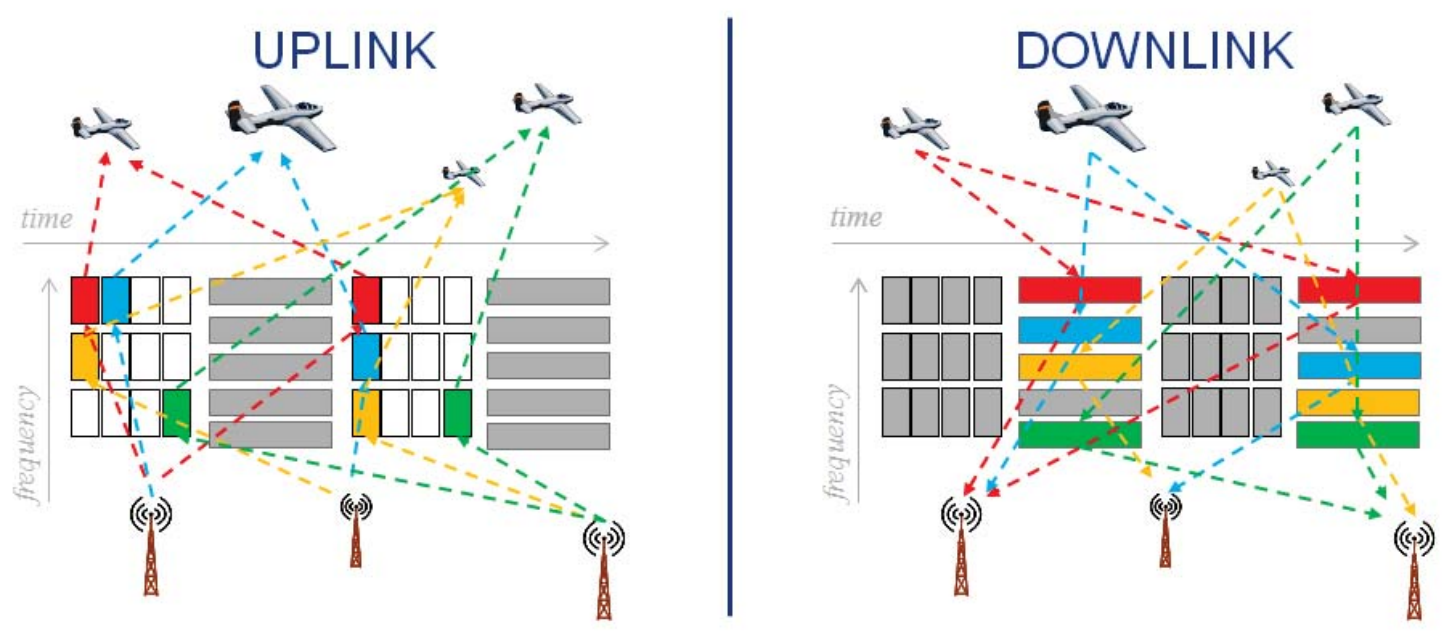


\section{GRC UAS Project - Communications Modeling and Simulation}

GRC UAS Communications - Opnet CNPC Gen1 Radio Model Validation

Gen1 Model Validation - Bench Tests

- Bench tests designed to validate protocol performance of the Gen1 radio models

- Tests performed with direct RF connection between 2 radios

- Test cases designed to exercise segmentation and queuing functions of the Gen1 waveform combined with the TDD/TDMA/FDMA transmission timing

- Tests validated total throughput and end-to-end delays through the radio for the test cases

Gen1 Model Validation - Flight Tests

- Flight tests used to validate RF performance of the Gen1 radio models

- Focused on signal propagation / received signal strength for the early model

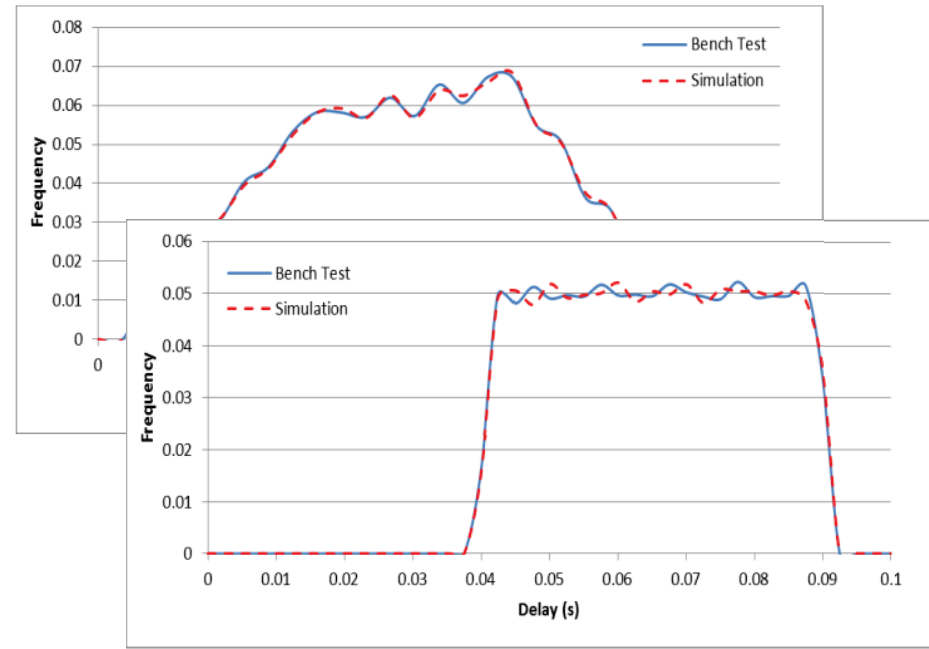

Bench Test End-End Delay Distribution Comparisons

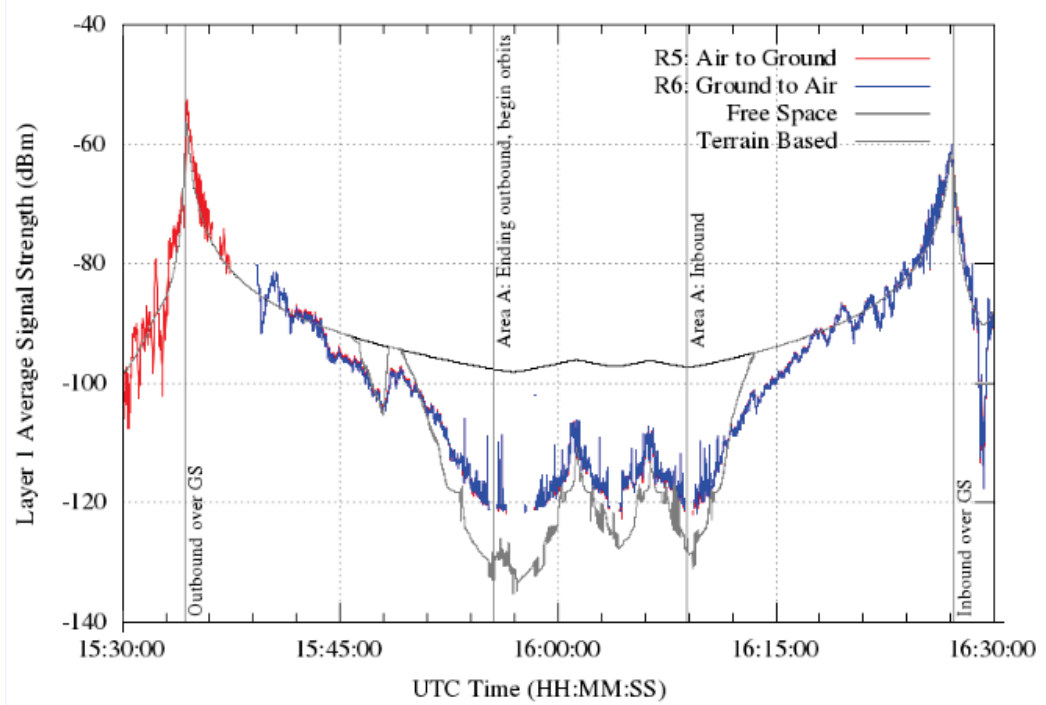

Flight Test Received Power Comparison 
GRC UAS Project - Communications Modeling and Simulation GRC UAS Communications - Opnet CNPC Gen2 Radio Model Test/Verification (to date)

\section{Gen2 Model Testing/Verification}

- Bench testing will again be used to examine protocol performance

- Tests designed to exercise priority between connections, queuing, and segmentation functions

- Additional tests will examine simple handoff functionality

- Focus on system throughput and delays

- Flight tests (scheduled for 4/2014) validate RF performance

- Received signal strength

- Link health metrics 
ACES - Base Application with Opnet providing CNPC Radio Models operating onboard UA

- Provides Air Traffic, NAS Operations, Aircraft Characteristics and Legacy NAS Comm Architecture

- Provides the UAS Comm Architecture component models for Relay and Non-Relay operations

- Provides equipage of UA and Piloted flights with onboard Voice/CPDLC Radios (Relay Arch)

- Provides VHF and CPDLC Ground Station infrastructure (Relay Arch)

- Provides ATC $\longleftrightarrow \rightarrow$ Pilot and ATC $\longleftrightarrow \rightarrow$ PIC Communication message sequences

- Uses Flight Data Sets (FDSs) to define number of UA and Piloted Flights flown in a simulation

\section{Opnet - Provides CNPC Radio Models to each UA and the CNPC Ground Infrastructure}

- Equips each UA with onboard CNPC radios running in Opnet Modeler

- Provides the CNPC Ground architecture for CNPC Radios operation in the NAS.

- Creates Instances of UAVs based on time-of-departure data from ACES

- Provides continuous UA CNPC link data streams for C2 uplink and Telemetry downlink

- Provides the integration of ATM messaging into CNPC Link for UAS Comm Relay operation

- Manages CNPC operations for the datalink using its own, independent GS Architecture 


\section{GRC UAS Project - Communications Modeling and Simulation} GRC UAS Communications - Large-scale Simulation Description (cont.)

\section{ACES-Opnet Interoperabilty}

- Use of an HLA/RTI application for overall simulation timing management

- Uses a common Trajectory Generator type to provide Flight State Data

- Allows aircraft position and other data to be common to both applications.

- Using Common Flight Data Sets to define UA and Piloted Flights in a simulation

- ACES uses All Flights (Piloted and UA).

- Opnet is provided with knowledge of only UA Flights from the ACES FDS

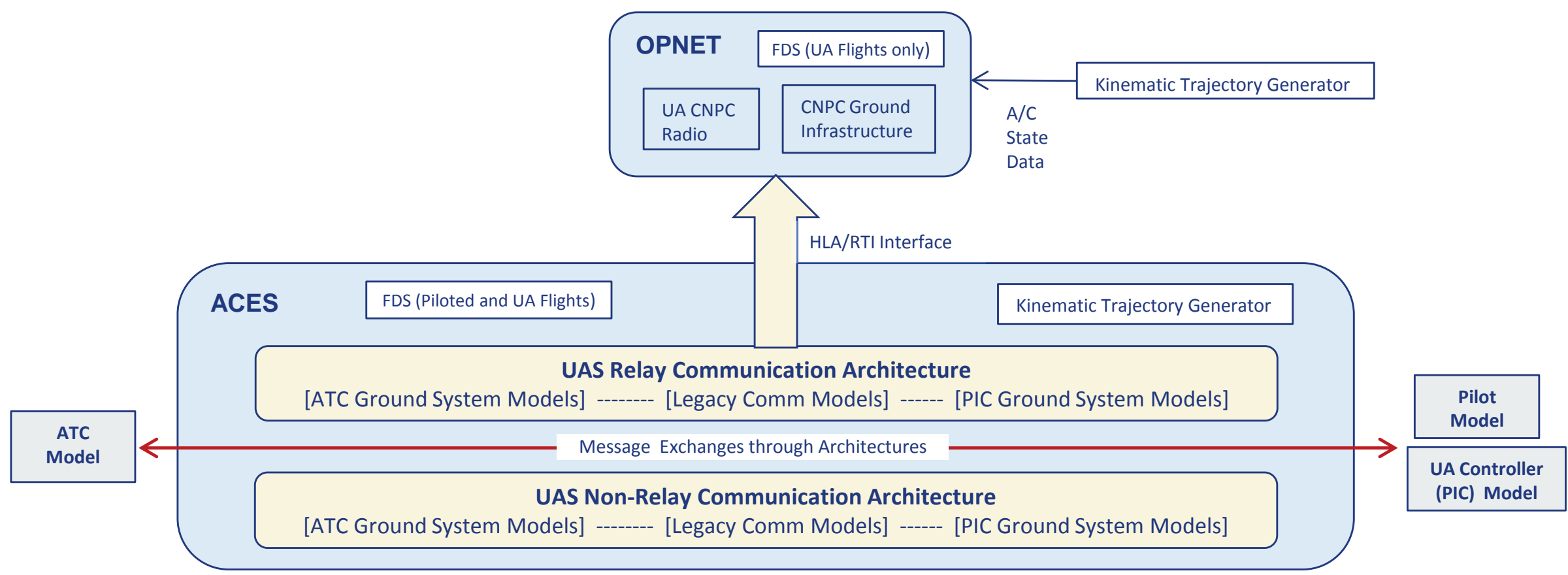




\section{GRC UAS Project - Communications Modeling and Simulation} GRC UAS Communications - Integrated Simulation Illustration

1. ACES synchronizes with OPNET over RTI for common timing operations

- New UA Aircraft Initiated by Flight Data Set

2. Aircraft locates nearby tower and establishes CNPC connection

3. Control and TLM traffic begins flowing between PIC and aircraft over CNPC link
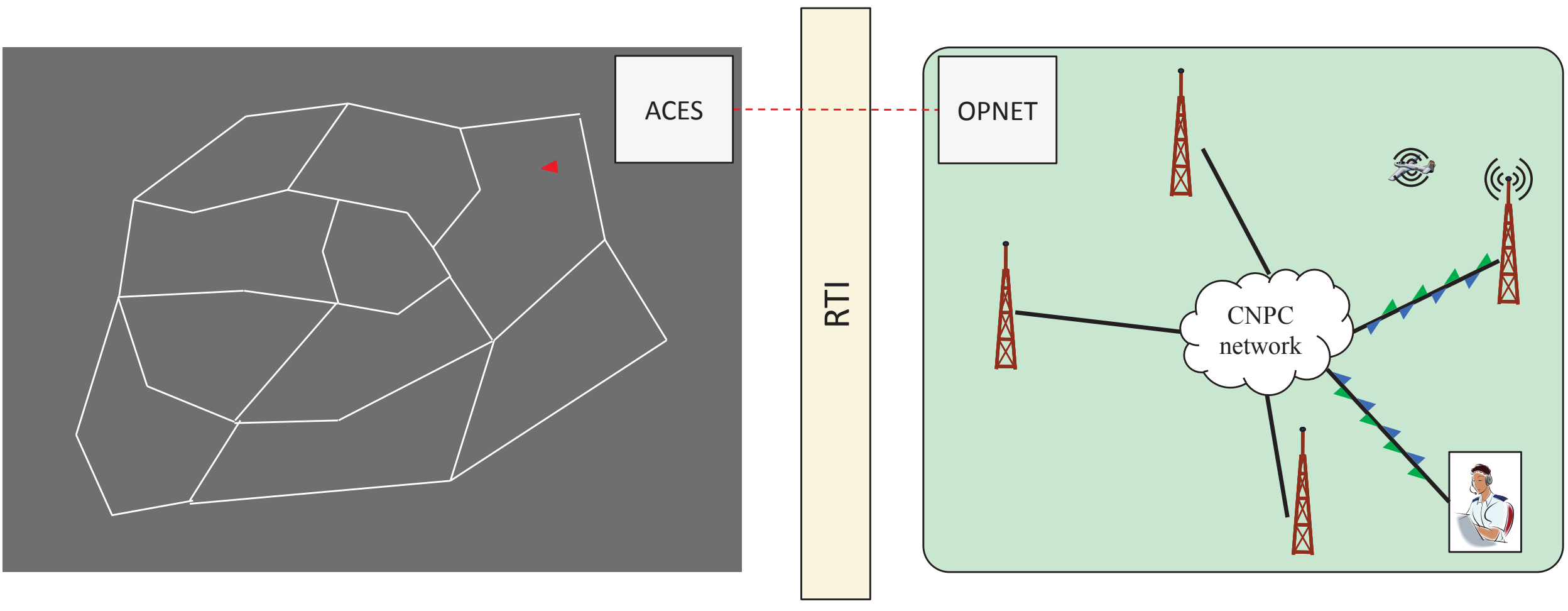
GRC UAS Project - Communications Modeling and Simulation GRC UAS Communications - Integrated Simulation Illustration (cont.)

4. Aircraft movement is synchronized between simulators (Common Trajectory Generators)

5. ACES generates voice or data message (e.g. in response to sector crossing)

6. Voice/data message handed-off to OPNET simulation for transfer over CNPC link

7. Message success indication returned to ACES when reception completed

(Timing reflects CNPC link delay)

8. ACES completes message delivery to recipient ACES model (e.g. PIC)

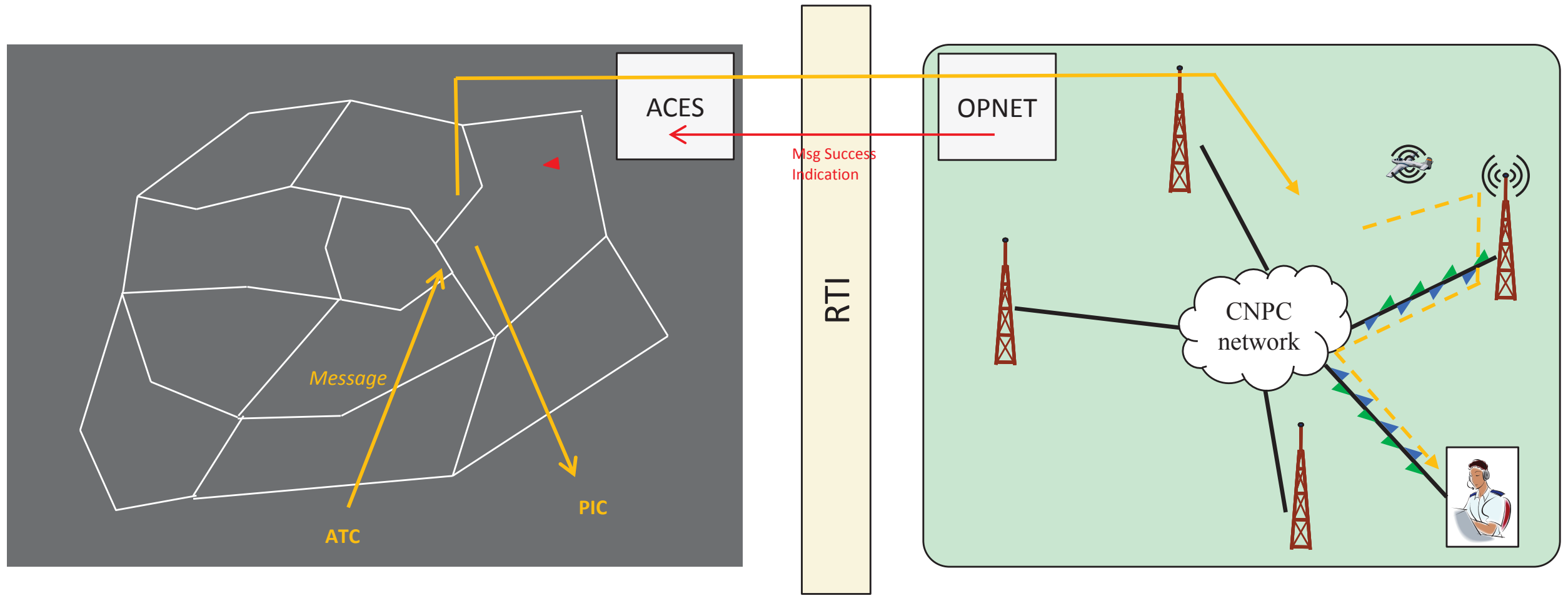


GRC UAS Project - Communications Modeling and Simulation GRC UAS Communications - Integrated Simulation Illustration (cont.)

9. As aircraft moves through the airspace:

- CNPC handoffs occur to new CNPC towers

- ATC site for ATM moves to each new responsible controller
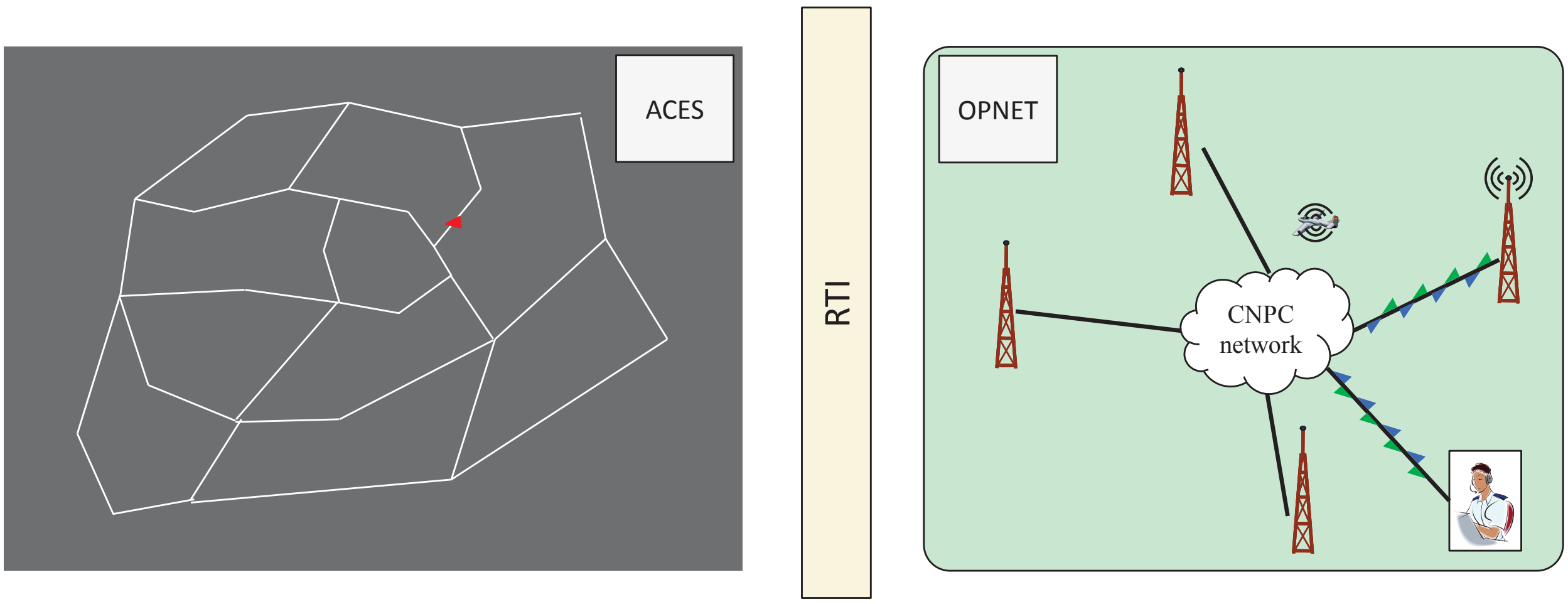
GRC UAS Project - Communications Modeling and Simulation

GRC UAS Communications - Large-scale Simulation Development Status

\section{Development Status}

- Completed integration of the Kinematic Trajectory Generator in Opnet

- Also developed an Opnet-side application for distributing per-flight state data to each instance of UA Aircraft

- Completed development of the Relay and Non-Relay Architectures in ACES including:

- ATC Ground Systems Models (Terrestrial FAA and DSP Network Models and Codec Models)

- Network Models and Codecs include configurability for fixed or statistical message delays

- PIC Ground Systems Models (Terrestrial CSP Network Model and PIC Codec Model)

- Network Models and Codecs include configurability for fixed or statistical message delays

- ATM Radio Model upgrades

- Added UAV VHF Radio Model functionality into VHF Model (for Relay Arch)

- Added UAV VDL2 Radio Model functionality into VDL2 Model (for Relay Arch)

- Comm Message Interface Model (for transferring msgs between ACES and Opnet over RTI)

- Completed Fed File Development for Message Hand-off iteractions

- Developed Opnet Interface for receiving and distributing messages to UA

- Completed new mechanism to define UA Aircraft to simulations from Flight Data Sets 
GRC UAS Project - Communications Modeling and Simulation

GRC UAS Communications - Large-scale Simulation Testing Status

\section{Testing Status}

- Tested/Verified the Opnet Kinematic Trajectory Generator

- Provided State Data to 1000+ UA Flights

- Tested end-to-end operation of both the Relay Architecture and Non-relay Architectures

- Verified ATM message transfers between all architecture model components for a combined UA and Piloted Flight Data Set of 750 Flights

- Verified timing configuration control mechanisms for ATC and PIC Ground Model components

- Verified operation and timing of the ACES-Opnet Comm Message Interface for:

- Correct interaction/message hand-offs with an ACES Opnet loopback (i.e. test) interface

- Correct interaction interoperability and message hand-offs with Opnet using HLA/RTI

- Verified equipage configuration capability for UA for use of Voice and/or Datalink ATM communications during phases of flight

- Incorporated and tested mechanism in ACES for designating UA Flights vs Piloted Flights based on Flight Data Set (FDS) Airline Name

- Verified operation to distinguish UAs in Large-scale simulations

- Verified operation to define which aircraft in a FDS are passed to Opnet KTG (UA Only) 
GRC UAS Project - Communications Modeling and Simulation

GRC UAS Communications - Large-scale Simulation Current Activity

Current Activity

- Preparing to Integrate first Opnet CNPC Radio Model into Large-scale simulations

- Upgrading ACES Version to Version 8.3

- Improved KTG Operation

- Improvements to accommodate shorter routes for UAV flights

- Upgrading ACES Voice Model for improved Message transmission (ATC/PIC/Pilot) wait-times

- Add improved Human Factor detection algorithm to the Voice Model to create more realistic Step-on performance in the VHF link.

- Takes into account the type/duration of delays expected in UA Relay and Non-relay architectures

- Currently running simulations and analyzing data for Large Flight Data Sets for Relay Architecture, CNPC link message traffic load characterization

- Integrate this characterization information into Opnet Modeler data generator models to more accurately load CNPC Voice service channel in detailed Opnet simualtions. 